\title{
Effect of Core/Shell Interface on Carrier Dynamics and Optical Gain Properties of Dual-Color Emitting CdSe/CdS Nanocrystals
}

Valerio Pinchetti, ${ }^{1}$ Francesco Meinardi, ${ }^{1}$ Andrea Camellini, ${ }^{2}$ Gianluca Sirigu, ${ }^{2}$ Sotirios Christodoulou, ${ }^{3}$ Wan Ki Bae, ${ }^{6}$ Francesco De Donato, ${ }^{3}$ Liberato Manna, ${ }^{3}$ Margherita Zavelani-Rossi, ${ }^{4,5}$ Iwan Moreels, ${ }^{3 *}$ Victor I. Klimov ${ }^{6 *}$ and Sergio Brovelli ${ }^{1^{*}}$

${ }^{1}$ Dipartimento di Scienza dei Materiali, Università degli Studi di Milano-Bicocca, Via Cozzi 55, IT20125 Milano, Italy

${ }^{2}$ Dipartimento di Fisica, Politecnico di Milano, P.zza Leonardo da Vinci 32, IT-20133 Milano, Italy

${ }^{3}$ Istituto Italiano di Tecnologia, Via Morego 30, IT-16163 Genova, Italy

${ }^{4}$ Dipartimento di Energia, Politecnico di Milano, Via Ponzio 34/3, IT-20133 Milano, Italy

${ }^{5}$ Institute for Photonics and Nanotechnologies IFN-CNR, P.zza Leonardo da Vinci 32, IT-20133 Milano, Italy

${ }^{6}$ Chemistry Division, Los Alamos National Laboratory, Los Alamos, New Mexico 87545, United States

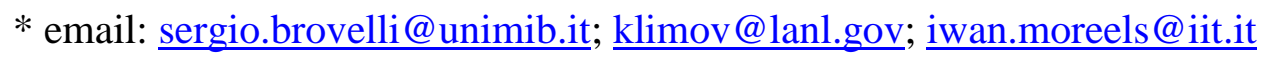



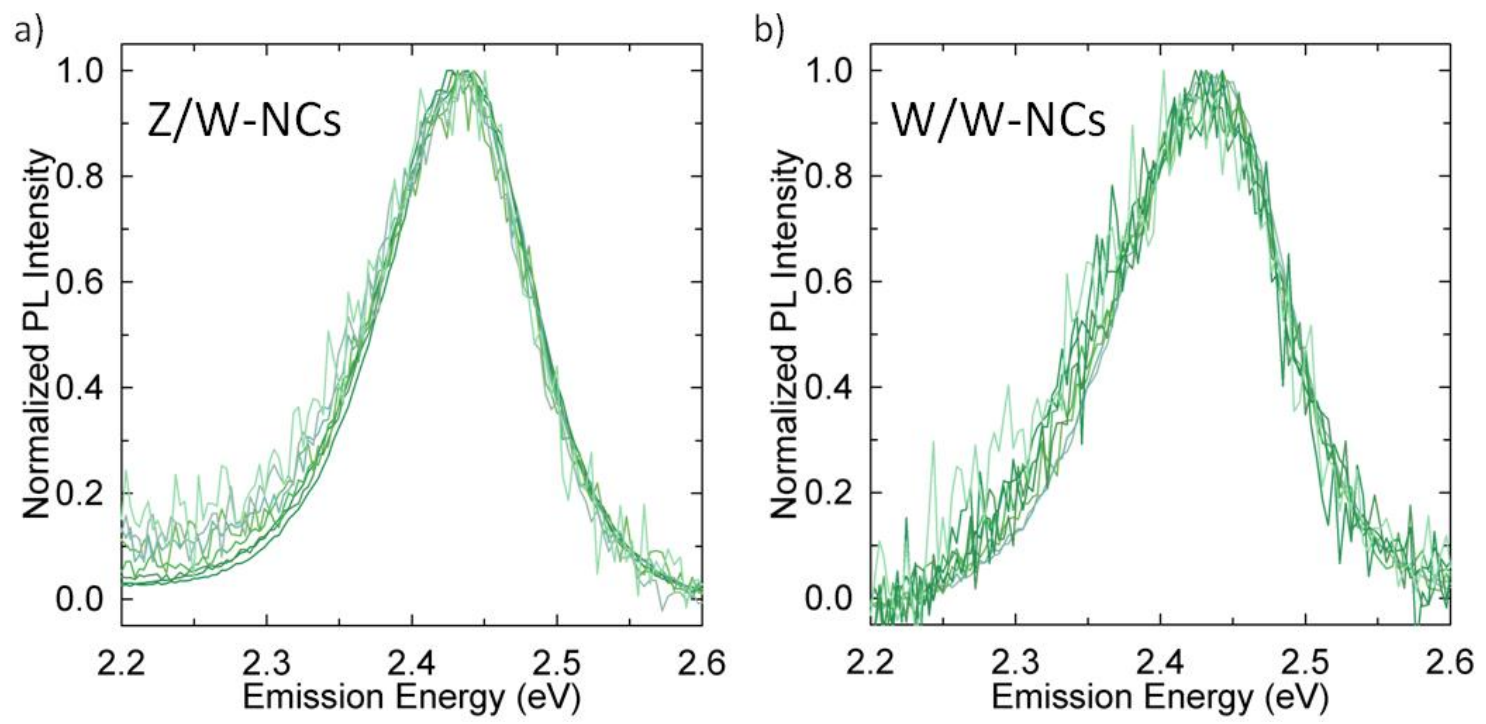

Supporting Fig.1 Normalized shell PL spectra of 1.5/8.5 CdSe/CdS DiB-NCs with (a) heterogeneous (Z/W-NCs) and (b) homogeneous (W/W-NCs) shell structures upon increasing excitation fluence $\left(1 \times 10^{-2} \mu \mathrm{J} / \mathrm{cm}^{2}<\Phi_{\text {exc }}<22 \mu \mathrm{J} / \mathrm{cm}^{2}\right.$, excitation energy $\left.3.1 \mathrm{eV}\right)$. 
Shifts of the CB and VB levels in quantum-confined CdSe NCs with respect to the band edges of bulk $\mathrm{CdSe}$

\begin{tabular}{|c|c|c|c|c|c|}
\hline Cryst. Phase & $\mathrm{R}[\mathrm{nm}]$ & $\delta \mathrm{E}_{\mathrm{CB}}[\mathrm{eV}]$ & $\delta \mathrm{E}_{\mathrm{VB}}[\mathrm{eV}]$ & Method & Ref. \\
\hline WZ & 1.5 & 0.65 & - & XAS & Lee et $a l .{ }^{I}$ \\
\hline WZ & 1.4 & 0.50 & -0.25 & $\mathrm{CV}$ & Markus et al. ${ }^{2}$ \\
\hline WZ & 1.4 & 0.55 & 0 & PES & Markus et al. ${ }^{2}$ \\
\hline $\mathrm{ZB}$ & 1.5 & 0.5 & -- & TB Theory & Lippens et al. ${ }^{3}$ \\
\hline $\mathrm{ZB}$ & 1.5 & 0.7 & -0.4 & FPC Theory & Wang et $a .^{4}$ \\
\hline $\mathrm{ZB}$ & 1.5 & 0.65 & -- & XAS & Meulenberg et al..$^{5}$ \\
\hline $\mathrm{ZB}$ & 1.5 & -- & -0.25 & CV & Meulenberg et al..$^{5}$ \\
\hline
\end{tabular}

Supporting Table.1 Confinement-induced shift of the conduction- $\left(\delta \mathrm{E}_{\mathrm{CB}}\right)$ and valence-band $\left(\delta \mathrm{E}_{\mathrm{VB}}\right)$ levels of CdSe NCs with radius $R=1.4-1.5 \mathrm{~nm}$ in either wurtzite (WZ) or zincblende (ZB) crystal phases with respect to the band edges of bulk CdSe.

CB and VB energy offsets between the zincblende (ZB) and wurtzite (WZ) phases of CdS

\begin{tabular}{c|c|c|c|c}
\hline Type of structure & $\Delta \mathrm{E}_{\mathrm{CB}, \mathrm{ZB} / \mathrm{wZ}}[\mathrm{meV}]$ & $\Delta \mathrm{E}_{\mathrm{VB}, \mathrm{ZB} / \mathrm{wZ}}[\mathrm{meV}]$ & Method & Ref. \\
\hline Bulk & 131 & 44 & DF Theory & Li et al. $^{6}$ \\
Bulk & 115 & 46 & FPC Theory & Wei et $^{7}$ \\
Superlattice & 70 & 30 & PP Theory & Bandić ${\text { et } a .^{8}}^{8}$ \\
Bulk & 73 & 31 & LDA Theory & Murayama et al.
\end{tabular}

Supporting Table.2 Conduction $\left(\Delta \mathrm{E}_{\mathrm{CB}}\right)$ and valence-band $\left(\Delta \mathrm{E}_{\mathrm{VB}}\right)$ energy offsets between the wurtzite (WZ) and zincblende (ZB) crystal phases of CdS.

\section{Table legend}

XAS = X-Ray Absorption Spectroscopy

$\mathrm{CV}=$ Cyclic Voltammetry

PES = Photoelectron Spectroscopy

TB Theory $=$ Tight-Binding Theory

FPC Theory $=$ First-Principles Calculation Theory

DF Theory $=$ Density Functional Theory

PP Theory $=$ Pseudopotential Theory

LDA Theory $=$ Local Density Approximation Theory 


\section{Band-edge energies (vs. vacuum) in $\mathrm{CdSe}(\mathrm{ZB}) / \mathrm{CdS}(\mathrm{ZB}) / \mathrm{CdS}(\mathrm{WZ})$ heterojunctions reconstructed from literature data for $\mathrm{CdSe} \mathrm{NCs}$, thin $\mathrm{CdS}$ layers and bulk $\mathrm{CdS}$.}

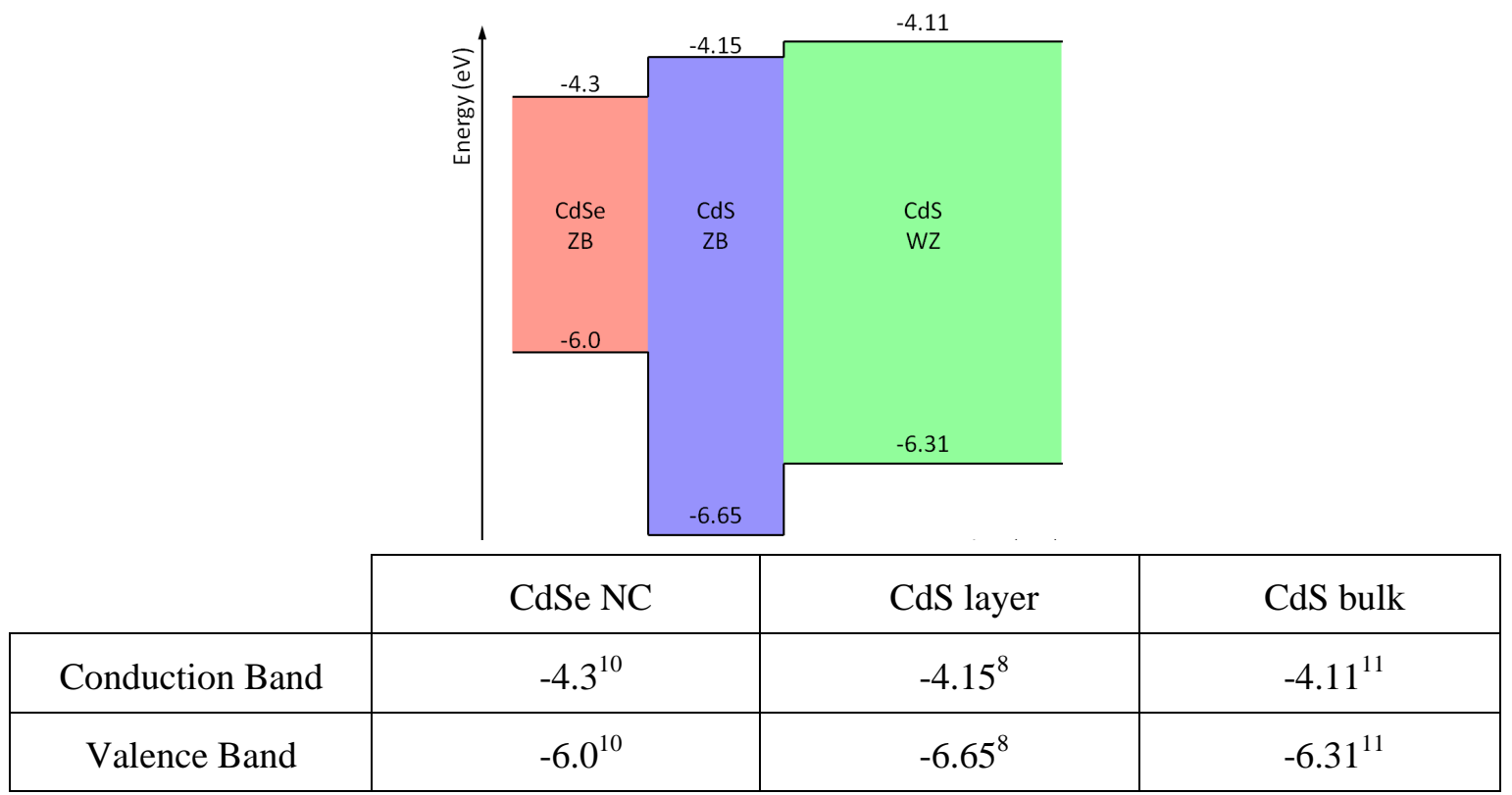

Supporting Table.3 Band-edge energies (vs. vacuum) in heterojunctions of $\mathrm{CdSe}(\mathrm{ZB}), \mathrm{CdS}(\mathrm{ZB})$, $\mathrm{CdS}(\mathrm{WZ})$ resembling the structure of $\mathrm{Z} / \mathrm{W}$ DiB-NCs studied in the present work.

In Supporting Table 1 we report the literature experimental and theoretical values of the conduction band $\left(\delta \mathrm{E}_{\mathrm{CB}}\right)$ and valence band $\left(\delta \mathrm{E}_{\mathrm{VB}}\right)$ shifts in $\mathrm{CdSe} N C$ s in both $\mathrm{ZB}$ and $\mathrm{WZ}$ crystal structures with respect the bulk energy gap due to quantum confinement effects. The confinement-induced energy shifts are much larger for the conduction band than for the valence band because of a much smaller effective mass of an electron with respect to a hole mass in II-VI semiconductors. In Supporting Table 2, we report literature data for the $\mathrm{CB}$ and VB energy offsets between the $\mathrm{ZB}$ and WZ phases of CdS. Finally, in Supporting Table 3, we report CB and VB energies of nanostructured and bulk materials that resemble the three different elements of our Z/W-NCs; these include ZB CdSe NCs, ZB CdS quantum well structures, and WZ bulk CdS. In all cases, the $\mathrm{CB}$ energies are essentially aligned, which explains why the electron wavefunction is delocalized over the entire DiB-NCs. This is also in agreement with theoretical calculations by Viswanatha and Sarma indicating a CB offset of $\leq 100 \mathrm{meV} .^{12}$ The electrostatic attraction by the core-localized hole gives rise to of a donor-like core exciton, in agreement with theoretical calculations by Shabaev and Efros. ${ }^{13}$ The WZ-CdS interlayer separating the $\mathrm{CdSe}$ core and the $\mathrm{CdS}$ shell, introduces a potential barrier in the valence band that exceeds the kinetic energy of bulk-like shell holes, thereby dramatically slowing down the rate of hole capture by core states. 


\section{Supporting References}

1. Lee, J. R. I.; Meulenberg, R. W.; Hanif, K. M.; Mattoussi, H.; Klepeis, J. E.; Terminello, L. J.; van Buuren, T. Experimental Observation of Quantum Confinement in the Conduction Band of CdSe Quantum Dots. Phys. Rev. Lett 2007, 98, 146803.

2. $\quad$ Markus, T. Z.; Wu, M.; Wang, L.; Waldeck, D. H.; Oron, D.; Naaman, R. Electronic Structure of CdSe Nanoparticles Adsorbed on Au Electrodes by an Organic Linker: Fermi Level Pinning of the Homo. $J$. Phys. Chem. C 2009, 113, 14200-14206.

3. Lippens, P. E.; Lannoo, M. Comparison between Calculated and Experimental Values of the Lowest Excited Electronic State of Small CdSe Crystallites. Phys. Rev. B 1990, 41, 6079-6081.

4. Wang, L.-W.; Li, J. First-Principles Thousand-Atom Quantum Dot Calculations. Phy. Rev. B 2004, $69,153302$.

5. Meulenberg, R. W.; Lee, J. R. I.; Wolcott, A.; Zhang, J. Z.; Terminello, L. J.; van Buuren, T. Determination of the Exciton Binding Energy in CdSe Quantum Dots. ACS Nano 2009, 3, 325-330.

6. Li, D.; Liu, Y.; Mata, M.; Magen, C.; Arbiol, J.; Feng, Y.; Xiong, Q. Strain-Induced Spatially Indirect Exciton Recombination in Zinc-Blende/Wurtzite Cds Heterostructures. Nano Res. 2015, 8, 30353044.

7. Wei, S.-H.; Zhang, S. B. Structure Stability and Carrier Localization in $\mathrm{CdX}(\mathrm{X}=\mathrm{S}, \mathrm{Se}, \mathrm{Te})$ Semiconductors. Phys. Rev. B 2000, 62, 6944-6947.

8. $\quad$ Bandić, Z. Z.; Ikonić, Z. Electronic Structure of $(\mathrm{Zn}, \mathrm{Cd})(\mathrm{S}, \mathrm{Se})$-Based Polytype Superlattices. Phys. Rev. B 1995, 51, 9806-9812.

9. Murayama, M.; Nakayama, T. Chemical Trend of Band Offsets at Wurtzite/Zinc-Blende Heterocrystalline Semiconductor Interfaces. Phys. Rev. B 1994, 49, 4710-4724.

10. Cheng, C.-T.; Chen, C.-Y.; Lai, C.-W.; Liu, W.-H.; Pu, S.-C.; Chou, P.-T.; Chou, Y.-H.; Chiu, H.-T. Syntheses and Photophysical Properties of Type-II CdSe/ZnTe/ZnS (Core/Shell/Shell) Quantum Dots. $J$. Mater. Chem. 2005, 15, 3409-3414.

11. Xing, C.; Zhang, Y.; Yan, W.; Guo, L. Band Structure-Controlled Solid Solution of Photocatalyst for Hydrogen Production by Water Splitting. Int. J. Hydrogen Energ. 2006, 31, 2018-2024.

12. Viswanatha, R.; Sarma, D. D. Effect of Structural Modification on the Quantum-Size Effect in II-VI Semiconducting Nanocrystals. Chem Asian J 2009, 4, 904-909.

13. Shabaev, A.; Rodina, A. V.; Efros, A. L. Fine Structure of the Band-Edge Excitons and Trions in CdSe/CdS Core/Shell Nanocrystals. Phys. Rev. B 2012, 86, 205311. 